\title{
Erratum: Immunohistochemical investigation of neuronal injury in cerebral cortex of cobra-envenomed rats
}

In the article entitled "Immunohistochemical investigation of neuronal injury in cerebral cortex of cobra-envenomed rats", DOI: 10.1590/S1678-91992004000100005, published in Journal of Venomous Animals and Toxins including Tropical Diseases, 10(1), 2004,

Page 1, where it was written:

RAHMY T.R. ${ }^{1}$; HASSONA I.A. ${ }^{2}$

${ }^{1}$ Zoology Department, Faculty of Science, Suez Canal University, Ismailia, Egypt; ${ }^{2}$ Biology Department, Faculty of Science, United Arab Emirates University, UAE.

It should be read:

RAHMY T.R. ${ }^{1}$; HASSOUNA I.A. ${ }^{2}$

${ }^{1}$ Zoology Department, Faculty of Science, Suez Canal University, Ismailia, Egypt; ${ }^{2}$ Zoology Department, Faculty of Science, Menoufia University, Egypt. 